\title{
Anemia aplásica
}

\section{Efreen H. Montaño-Figueroa ${ }^{1 *}$ y David Gómez-Almaguer ${ }^{2}$}

${ }^{1}$ Pabellón de Hematología, Hospital General de México Dr Eduardo Liceaga, Ciudad de México; ${ }^{2}$ Servicio de Hematología, Hospital Universitario Dr José E. González, Universidad Autónoma de Nuevo León, Monterrey, N.L. México

\section{Resumen}

La pandemia de enfermedad por coronavirus 2019 (COVID-19) ha cambiado la perspectiva médica para el tratamiento de no solo de enfermedades hematológicas, sino en general de la medicina. Respecto a la anemia aplásica ( $A A)$, principalmente la muy severa, en la que el paciente se presenta con menos de 200 neutrófilos absolutos, el riesgo de infección potencialmente mortal es alta y el inicio de terapia inmunosupresora también representa un riesgo, al menos temporal, para COVID-19. Se ha recomendado incluso aplazar el trasplante de células progenitoras hematopoyéticas en muchos pacientes para evitar un contagio. Una inmunosupresión moderada preferentemente ambulatoria que incluya agentes trombomiméticos es la opción terapéutica en tiempos de la pandemia actual. En esta revisión se enlistan las recomendaciones internacionales y nacionales respecto al tratamiento y seguimiento de pacientes con $A A$ con base en experiencias de países que ya han pasado por esta emergencia sanitaria.

PALABRAS CLAVE: Anemia aplásica. Terapia inmunosupresora. Eltrombopag. COVID-19.

\section{Aplastic anemia}

\begin{abstract}
Medical practice in general has changed due to coronavirus disease 2019 (COVID-19) pandemic. Some hematologic diseases require immunosuppresive therapy placing patients at high risk of infection, including severe acute respiratory syndrome coronavirus 2 (SARS-CoV-2). Aplastic anemia (AA) especially the very severe type in which the count of absolute neutrophils is less than $200 / \mu l$ is a life-threatening condition. Although bone marrow transplant is a potential curative treatment, it should be delayed temporally in order to prevent a contagion. Hospitalization may expose patients to infection, thus an ambulatory immunosuppression with oral cyclosporine and thrombopoietin agonist should be an adequate option. This work reviews international and national treatment recommendations and follow-up of patients with AA based on experiences from countries that have already faced this health emergency.
\end{abstract}

KEY WORDS: Aplastic anemia. Immunosuppressive therapy. Eltrombopag. COVID-19.

\section{Introducción}

La anemia aplásica (AA) adquirida se inicia cuando ocurre una activación y expansión oligoclonal de células T que conllevan daño autoinmune de la célula progenitora hematopoyética. Las citopenias secundarias incrementan el riesgo de hemorragias e infecciones graves que pueden ser fatales ${ }^{1}$. Para el diagnóstico se requiere excluir otras causas de pancitopenia tales como infección, ingesta de medicamentos, deficiencias nutricionales e incluso enfermedades reumatológicas, además de un resultado de biopsia de hueso en el cual
Correspondencia:

*Efreen H. Montaño-Figueroa

E-mail: herrefreen@ hotmail.com

0016-3813/@ 2021 Academia Nacional de Medicina de México, A.C. Publicado por Permanyer. Este es un artículo open access bajo la licencia CC BY-NC-ND (http://creativecommons.org/licenses/by-nc-nd/4.0/).
Fecha de recepción: 09-09-2020

Fecha de aceptación: 09-02-2021
Gac Med Mex. 2021;157(Supl 3):S52-S54

Disponible en PubMed

www.gacetamedicademexico.com 
haya hipocelularidad y se excluya infiltración tumoral y presencia de reticulina. La severidad de la AA depende del porcentaje de celularidad en médula ósea, así como de la cuenta inicial de plaquetas, neutrófilos y reticulocitos ${ }^{2}$.

Hoy en día el único tratamiento curativo para la $A A$ es el trasplante de células progenitoras hematopoyéticas (TCPH), procedimiento que debe realizarse lo más tempranamente posible después del diagnóstico para garantizar el éxito. Otra opción de tratamiento es la inmunosupresión con globulina antitimocito (ATG), ciclosporina $A$ o alemtuzumab, principalmente para pacientes que no tienen un donante de médula ósea compatible disponible ${ }^{3}$. Los riesgos de recaída o un escape de hematopoyesis clonal hacia una hemoglobinuria paroxística nocturna, mielodisplasia o leucemia aguda siguen siendo la principal preocupación con esta terapia ${ }^{4}$. La adición de agentes trombomiméticos como eltrombopag a la terapia inmunosupresora ha mostrado un incremento en las respuestas tanto completas como parciales en un $88 \%$ a seis meses y un $40 \%$ a dos años de seguimiento, y sobre todo representa para muchos pacientes una terapia puente al $\mathrm{TCPH}^{5}$.

\section{Experiencia mundial}

La pandemia por coronavirus 2 del síndrome respiratorio agudo grave (SARS-CoV-2) es actualmente una emergencia en salud; la exposición al virus de los pacientes que reciben tratamiento antineoplásico o terapia inmunosupresora incrementa considerablemente el riesgo de contraer esta cepa de coronavirus. Es por ello que, con base en experiencias de países afectados por esta enfermedad, se han dado algunas recomendaciones médicas para evitar o disminuir la probabilidad de infección.

\section{Recomendaciones emitidas por la Sociedad Europea de Trasplante de Células Progenitoras Hematopoyéticas ${ }^{6}$}

La recomendación principal es que el paciente debe mantenerse en casa el mayor tiempo posible hasta que las autoridades sanitarias de cada país notifiquen que es seguro salir a la calle. El asistir a hospitales aumenta el riesgo de infección, tanto por otros pacientes como por el mismo personal de salud que pueden ser portadores asintomáticos. Deben mantenerse las salas de hospitalización para pacientes de AA libre de enfermedad por coronavirus 2019 (COVID-19), para ello a todos los pacientes ingresados debe realizarse una prueba de reacción en cadena de la polimerasa para SARS-CoV-2 y debe asignarse una sala especial para aquellos positivos; esto aplica también para área de TCPH y la unidad de terapia intensiva (UTI). En todo momento recomendar las acciones para disminuir el riesgo: uso de cubrebocas, lavado de manos y distanciamiento social ${ }^{6,7}$.

\section{Trasplante de células progenitoras hematopoyéticas}

Siempre que el estado de la enfermedad lo permita debe posponerse el TCPH hasta que la situación de contingencia esté controlada o haya suficiencia en la disponibilidad de camas en la UTI. En casos de anemia aplásica muy severa (menos de 200 neutrófilos) no se debe retrasar el TCPH, debe realizarse prueba de reacción en cadena de la polimerasa para SARS-CoV-2 tanto a paciente como al donante, y solo admitir a pacientes con prueba negativa. Deben aplicarse los esquemas de acondicionamiento, profilaxis de enfermedad injerto contra hospedero e inmunosupresión postTCPH de cada centro hospitalario; no es aconsejable realizar cambios o disminuir dosis recomendadas.

\section{Terapia inmunosupresora}

Cada paciente debe considerarse de forma individualizada para el inicio del tratamiento. Aunque no hay información suficiente para determinar la asociación de quienes reciben medicamentos depletores de linfocitos T, es conocido que su utilización incrementa el riesgo de infecciones virales en general y esto aplicaría para el coronavirus, por ello debe evitarse el uso de ATG o alemtuzumab. En caso ineludible para inicio de inmunosupresión, tal es el caso de pacientes con citopenias muy severas y que tienen riesgo de complicaciones mortales, debe realizarse prueba de reacción en cadena de la polimerasa para SARS-CoV-2 y solo iniciar corroborado un resultado negativo. Preferentemente optar por inmunosupresión moderada y que pueda llevarse en casa, como ciclosporina; el adecuado nivel sérico del fármaco es crucial para lograr la eficacia, por ello no debe obviarse realizar cuantificación de niveles séricos, especialmente los primeros seis meses desde el inicio. Para aquellos pacientes que ya ingerían ciclosporina antes de la pandemia, la dosis debe mantenerse. Si económicamente es factible, es recomendable agregar agentes trombomiméticos a la inmunosupresión oral que, aunque no tienen un efecto inmediato, 
eventualmente disminuyen el riesgo de citopenias severas (anemia/plaquetopenia) y dan oportunidad al paciente para aislamiento domiciliario ${ }^{8}$.

\section{Recaída o evolución clonal}

Para pacientes en recaída deben seguirse las mismas pautas médicas que pacientes recién diagnosticados, de igual forma optar por el tratamiento que el paciente pueda consumir en casa, como el eltrombopag y la ciclosporina. En caso de evolución clonal, en especial hacia Hemoglobinuria Paroxística Nocturna (HPN), debe posponerse el inicio de eculizumab o ravulizumab hasta término de la emergencia sanitaria. En pacientes evolucionados a leucemia aguda mieloide no debe haber restricción alguna para inicio de tratamiento quimioterápico, a menos que el paciente tenga sintomatología de infección por SARS-CoV-2.

\section{Terapia de soporte}

Disminuir la frecuencia de hospitalización para transfusión y solo prescribir a aquellos pacientes con hemoglobina por debajo de $7.0 \mathrm{~g} / \mathrm{dl}$ y recuento de plaquetas menor a 10,000. Si el paciente clínicamente amerita transfusión debe hacerse de forma ambulatoria y en caso posible en domicilio.

En relación con los factores estimulantes de granulocitos, debe valorarse cada caso individualmente, no se indican de forma sistemática. La recomendación en pacientes con COVID-19 es para minimizar o evitar infecciones bacterianas sobreagregadas.

\section{Recomendaciones nacionales}

Las recomendaciones internacionales aplican en nuestro país. Sin embargo, por razones económicas es posible que muchos centros o pacientes no tengan acceso a medicamentos como el eltrombopag, el cual debe administrarse a dosis altas en casos de AA. El uso de andrógenos sigue vigente y es especialmente útil en casos leves o moderados, se pueden combinar con ciclosporina. El danazol es una opción ideal, si no se consigue, otro andrógeno como la mesterolona puede ser administrado $0^{9,10}$.

\section{Conclusiones}

Los pacientes con AA muy grave o severa deben proceder rápidamente al trasplante, previa documentación de estar libres de COVID-19. Esto aplica también a su donador. En otros casos menos graves el uso de ciclosporina en combinación con eltrombopag es ideal; en casos seleccionados puede usarse danazol o mesterolona si el eltrombopag no está al alcance del paciente. Deben fomentarse las medidas de higiene, aislamiento y hasta lo posible la terapia ambulatoria o en domicilio.

\section{Conflicto de intereses}

Los autores declaran no tener conflictos de intereses alguno con el tema de la publicación.

\section{Financiamiento}

No se recibió financiamiento ni patrocinio por ninguna organización o institución.

\section{Responsabilidades éticas}

Protección de personas y animales. Los autores declaran que para esta investigación no se han realizado experimentos en seres humanos ni en animales.

Confidencialidad de los datos. Los autores declaran que en este artículo no aparecen datos de pacientes.

Derecho a la privacidad y consentimiento informado. Los autores declaran que en este artículo no aparecen datos de pacientes.

\section{Bibliografía}

1. Young NS. Aplastic anemia. N Engl J Med. 2018;379:1643-56.

2. Brodsky RA, Jones RJ. Aplastic anaemia. Lancet. 2005;365:1647-56.

3. Bacigulapo A. How I treat acquired aplastic anemia. Blood. 2017;129:1428-36.

4. Ogawa S. Clonal hematopoiesis in acquired aplastic anemia. Blood. 2016;128:337-48

5. Townsley DM, Scheinberg P, Winkler T, Desmond R, Dumitriu B, Rios O, et al. Eltrombopag added to standard immunosuppression for aplastic anemia. N Engl J Med. 2017;376:1540-50.

6. EBMT Guidelines 2020 [Internet]. European Society for Blood and Marrow Transplantation. Disponible en: www.ebmt.org

7. When to Quarantine. Stay home if you might have been exposed to COVID-19 [internet] CDC. [12 marzo 2021]. Disponible en:https://www. cdc.gov/coronavirus/2019-ncov/prevent-getting-sick/

8. Ecsedi M, Lengline É, Knol-Bout C, Bosman P, Eikema DJ, Afanasyev B, et al. Use of eltrombopag in aplastic anemia in Europe. Ann Hematol. 2019;98(6):1341-50.

9. Jaime-Pérez JC, Colunga-Pedraza PR, Gómez-Ramírez CD, Gutiérrez-Aguirre CH, Cantú-Rodríguez OG, Tarín-Arzaga LC, et al. Danazol as first-line therapy for aplastic anemia. Ann Hematol. 2011;90(5):523-7.

10. Khurana $H$, Malhotra $P$, Sachdeva MU, Varma $N$, Bose $P$, Yanamandra $U$, et al. Danazol increases $T$ regulatory cells in patients with aplastic anemia. Hematology. 2018;23(8):496-500. 